\title{
Who is Becoming Autistic and Why?
}

\author{
Maria Kuman* \\ Holistic Research Institute, USA \\ *Corresponding Author: Maria Kuman, Holistic Research Institute, USA.
}

Received: September 16, 2019; Published: October 10, 2019

DOI: 10.31080/ASNH.2019.03.0495

The symptoms and manifestation of autism are very similar to the symptoms and manifestation of mercury poisoning claims Bernard Rimland, Director of the Autism Research Institute, who consulted on the movie 'Rain Man'. He also claims that 'mercury is extraordinary toxic in small amounts, but some people are amazingly susceptible to minute amounts of mercury' [1]. Thus, the amount of mercury in the vaccines may not be safe for everybody. This article is about who are these extra-sensitive people.

We are not only body. We are body and nonlinear electromagnetic field (NEMF), which we call biofield and photograph with Kirlian photography as aura. Biofield is not the right name for it because everything material is a material body and NEMF [2]. The author had proved with measurements that the crystals NEMF influences the human NEMF and can be used to balance the energy of our body, i.e. to heal [3].

Valerie Hunt [4] studied for many years the human biofield (NEMF). Her research showed that the major frequency of the human NEMF could vary dramatically from individual to individual. The majority of people have NEMF with major frequency $200 \mathrm{~Hz}$. However, people that are clairvoyant have NEMF with major frequency between $400 \mathrm{~Hz}$ and $800 \mathrm{~Hz}$. People that can travel in other dimensions have NEMF with major frequency between $800 \mathrm{~Hz}$ and $900 \mathrm{~Hz}$. Some people can even have NEMF with major frequency thousands of $\mathrm{Hz}$.

The higher is the major frequency of the NEMF, the higher is the sensitivity of the individual - emotional sensitivity, stress sensitivity, and drug sensitivity. We said that the majority of people have NEMF with major frequency $200 \mathrm{~Hz}$. What do we mean by majority? The answer is $90 \%$. So, what is considered safe amount of mercury for the $90 \%$ of people may not be safe for the $10 \%$ with higher frequency NEMF and higher sensitivity. But let us go step by step.

I spent 40 years measuring the weak NEMF. I had to develop sensitive enough equipment to be able to measure it because it is 1,000 times weaker than the biocurrents of the body. I found that this weak NEMF rules and regulates the functioning of all our organs. It is doing this with the information it carries, not with its weak currents. Since we don't have awareness for the functioning of our organs, the functioning of our organs is subordinated to the Subconscious.
However, all our organs are holographically represented on the surface of the skin: on our palms, feet, ears, and the irises of our eyes. The acupuncturists know that they can treat the points of the sick organ on any of these representations with equal success [5]. Therefore, we have holographic representations of the organs on the surface of the body to tell us that our organs are ruled and regulated on holographic principle [6]. Since we use lasers to create holographic images and lasers are coherent waves with certain frequency, waves must regulate the functioning of our organs.

In 1983, the author predicted with nonlinear mathematical model that each acupuncture treatment must generate electric impulse and wave [7]. The theoretically predicted waves were experimentally found one year later [8]. It was found that waves propagate along he acupuncture meridians all the time, but when a point of the meridian is treated with acupuncture, a wave is generated, which modifies the constantly running waves of our NEMF.

Russian scientists investigated Bronnikov, who could see with his mind, and found [9] that he could see 3-dimensional holographic image of the object in front of him (with his mind) when he was blindfolded and there was no signal propagating from his eyes to his brain. Who are the people that can see with their minds? These are the same high-sensitivity people (emotional, stress and drug sensitive) with high major frequency of their NEMF.

Thus, if we use on these highly sensitive people vaccines with the same amount of mercury, which we use for low-sensitive people, the sensitive people will become damaged because the amount of mercury, which the low-sensitive people can tolerate, will be too much for them. And these highly emotional individuals are very intuitively creative [10]. So, with the high amount of mercury in our vaccines, we are disabling the most creative people, on whom the fast development of our civilization depends.

From hypnosis, which puts the conscious to sleep to access the subconscious, we know that hypnotized people can calculate 10,000 times faster [11]. So, sophisticated quantum computer must operate in the Subconscious with the waves of our NEMF. Since, this is in the Subconscious, we don't have conscious awareness of it. However, the fact that people with high major frequency of their NEMF (400 to $800 \mathrm{~Hz}$ ) are clairvoyant means that they can 
use their Quantum Computer, which allows quantum jumps to the future and the past [12]

Also, in the film 'Rain Man', the mentally disabled man (with poorly functioning brain) could predict things or calculate with high speed, which indicate ability to use the Quantum Computer of the Subconscious. Mercury damages the neuronal connections in certain areas of the brain, which is considered to be the basis of the erratic autistic behavior. It damages mostly the emotionally sensitive individuals with high sensitivity to drugs, but we should do everything to eliminate or drastically reduce the amount of mercury for all our children.

Considering the damage, which mercury can do to the brain, I think the amount of mercury in the vaccines needs to be lowered at least 200 times or totally eliminated. Presently, the total amount of mercury in the vaccines is 200 times more than the amount that is considered safe by the CDC standards. The amount of mercury in the vaccines, which was considered safe, was calculated when the vaccines were 3 to the age of 6 and now they are 22 to the age of 2 . The best thing is to find substitute for the mercury because the number of vaccines will continue to grow.

\section{Bibliography}

1. B Rimland. "Vaccines, Autism, and Childhood Disorders" (2003)

2. M Kuman. "How Was the Material World Created? Origin of NEMF". Open Access Journal of Mathematical and Theoretical Physics 2 (2019).

3. M Kuman. "Effect of Crystals on the Body's NEMF". Journal of Natural and Ayurvedic Medicine 2 (2018).

4. Valerie Hunt. www.biofieldenergy.org

5. M Kuman. "Modern Aspects of Ancient Acupuncture, Health and Happiness Books" (1997).

6. M Kuman. "Holographic (Quantum) Ways of Seeing, Hearing, Smelling and Memorizing and How to Use Them for Diagnosis". Research in Medical and Engineering Sciences 5 (2018).

7. M Kuman. 8th World Congress on Acupuncture, Sofia (1983).

8. A Eory. "Waves Propagating along the Acupuncture Meridians Detected". Acupuncture and Electro-Therapeutic Research 9 (1984): 217.

9. M Kuman. Quantum Mind and Quantum Growth, Health and Happiness Books (2015).

10. M Kuman. "Full Intuitive Creativity Is a Coherent Work of the Quantum Computer of Our Subconscious and the Digital Computer of Our Conscious". MOJ Proteomic and Bioinformatics 7 (2018).
11. M Kuman. "Quantum Physics, Alternative Medicine, and Spirituality”. Global Journal of Medical Research 19 (3) 2019.

12. M Kuman. "The Quantum Computer in the Subconscious - a New Dimension". International Journal of Complimentary and Alternative Medicine 11 (2018).

Volume 3 Issue 11 November 2019

(C) All rights are reserved by Maria Kuman. 